\title{
An innovative short-term congestion management algorithm for the Italian subtransmission grid: the Zonal-EMS Demo of the OSMOSE Project
}

\author{
Luca Orrù ${ }^{*}$, Giuseppe Lisciandrello*, Stefano Gliozzi ${ }^{\dagger}$, Marco De Ieso ${ }^{\dagger}$, Antonello Aita ${ }^{\ddagger}$, \\ Riccardo Vignali ${ }^{\S}$ and Guido Coletta ${ }^{\S}$ \\ *Innovation Factory System Operator, Terna S.p.A., Rome, Italy \\ Email: \{luca.orru, giuseppe.lisciandrello\} @ terna.it \\ ${ }^{\dagger}$ Technology division, IBM Italy \\ Email: \{stefano_gliozzi, marco.deieso\}@it.ibm.com \\ ${ }^{\ddagger}$ Businses Services division, IBM Italy \\ Email: antonello.aita@ibm.com \\ $\S$ Department of Generation Technologies and Material \\ Ricerca sul Sistema Energetico S.p.A., Milan, Italy \\ E-mails: \{riccardo.vignali, guido.coletta\}@rse-web.it
}

\begin{abstract}
Power grids are getting through great transformations principally driven by massive Renewable Energy Sources penetration. Therefore, Transmission Systems Operators are currently working on building know-how to exploit new flexibility from this new class of generators installed throughout the system. In this context, the European Commission project OSMOSE aims at developing and testing a new Zonal-Energy Management System in order to coordinate innovative resources for congestion management in transmission and sub-transmission power grids, which is going to be tested in a portion of the sub-transmission system in the South of Italy. This paper describes the main architecture of the proposed Energy Management System and the first insights on the ongoing experimentation.
\end{abstract}

Index Terms-power system, energy management system, dynamic thermal rating, demand side response, optimal power flow

\section{NOMENCLATURE}

$\mathcal{T}$ set of indices corresponding to the time-steps

$\mathcal{F} \quad$ set of offers that can be activated in $\mathcal{T}$

$\mathcal{F}^{p} \quad$ set of past offers already activated in $\mathcal{T}$

$\mathcal{N} \quad$ set of buses

$\mathcal{N}_{g} \quad$ set of programmable generator buses

$\mathcal{N}_{b} \quad$ set of boundary buses

$\mathcal{N}^{l} \quad$ set of load buses

$\mathcal{N}_{e} \quad \mathcal{N}^{e}:=\mathcal{N}^{g} \cup \mathcal{N}^{l} \cup \mathcal{N}^{b}$

$\mathcal{L} \quad$ set of indices corresponding to the lines

$\Delta \hat{I}_{(i, j), t}^{d} \quad$ DTR overloading limit on line $(i, j) \in \mathcal{L}$ at time-step $t \in \mathcal{T}$

$I_{(i, j), t}^{u}$ static current upper bound on line $(i, j) \in \mathcal{L}$ at time-step $t \in \mathcal{T}$

This work has been funded by the European Commission project "OSMOSE-Optimal System-Mix of Flexibility Solutions for European Electricity", (Programme: H2020-EU.3.3.4. Topic: LCE-04-2017; Grant agreement ID: 773406).
Imaginary part of the Admittance matrix Active power injected at each bus Voltage angles at each bus penalty for each p.u. of active Power deviating from the program at each generation bus penalty for each p.u. of active Power deviating from the program at each boundary bus penalty term to guarantee that $I_{(i, j)}=\left|P_{(i, j)}\right|$. penalty term per each p.u. current, in any line, with larger than the static limit, but smaller than the DTR limit

penalty term to allow model completion even if there is a congestion penalty term for the activation of DSR offers penalty term to allow model completion even in case of heavy unbalance between generation and load

fixed cost associated to the offer $f$ variable cost associated to the offer $f$ activation signal of the offer $f$ module of the current above the maximum line limit (if DTR line, it will be the DTR defined limit) on line $(i, j)$ and time-step $t$ module of the current above the static limit but below the DTR limit on line $(i, j)$ and timestep $t$ difference of active power with respect to program $\hat{P}_{k, t}$ at bus $k \in \mathcal{N}^{g}$ and time-step $t$ difference of active power with respect to $\hat{P}_{k, t}^{b}$ at bus $k \in \mathcal{N}^{b}$ and time-step $t$ difference of active power with respect to pro- 
$E_{k, t}$

gram $\bar{P}_{k, t}$

slack in active power at bus $k$, defined for the set of buses $k \in \mathcal{N}$ such that $k$ is a load, or a generator or a boundary bus

\section{INTRODUCTION}

\section{A. Motivation and Literature Review}

In the last two decades, the power system industry has experienced deep transformations. The large diffusion of Variable Renewable Energy Sources (VRES), the deregulation of the electricity markets and the always increasing demands for energy at affordable prices have profoundly changed the way the electricity networks are planned and operated [1]. Transmission System Operators (TSOs) all around the world started to observe increasing levels of congestion in the transmission networks that must be managed in order to guarantee the system adequacy, as well as stable and secure operation.

The congestion management and mitigation techniques are usually classified as long-term and short-term strategies. Long term congestion management methods are based on optimal network expansion programs, which have effects on time horizons in the order of decades [2]. These techniques mainly lead to the construction of new interconnections [3], [4] and to the optimal placement of phase-shift and on-load tap changer transformers, FACTS devices [5], [6], etc., which can actually modify the power flows on the AC network. On the other hand, short-term congestion management methodologies aim at solving congestion as they occur through the implementation of market-based and non market-based policies. The simplest of these techniques are based on generators re-dispach, which allows to modify the power flows in the AC network through the coordinated operation of generators in the grid [7]-[9]. Another common way to solve congestion is through RES curtailment, which often are the main cause of congestion in modern transmission grids [10]. Furthermore, aggregated Demand Side Response (DSR) resources can be used for congestion management as they can modulate their power consumption with respect to a baseline based on price signal or participation to specific ancillary services provision markets [11]. Other short-term congestion dismantling techniques are based on the reconfiguration of existing networks, e.g. by changing the position of taps in transformers, network configuration or optimally dispatching flexibility sources offered by FACTS devices [12]. Another option for TSOs to solve congestion is to apply market distortion policies that encourage or discourage production and consumption such that energy does not flow on critical infrastructures, e.g. market splitting, local marginal prices, zonal prices and so on. Finally, in recent years the application of Dynamic Thermal Rating (DTR) techniques [13] has been demonstrated to effectively contribute to congestion management by unlocking additional capability margins based on the real-time exploitation of critical infrastructure thermal state (e.g. overhead lines, power cables, power transformer, etc.). Indeed, the integration of DTR techniques into advanced optimization frameworks, known as Weather Condition-based
Optimal Power Flow (W-OPF) or Electro-Thermal OPF (ET$\mathrm{OPF}$ ), allows to reliably enhance the congestion management by extracting actionable intelligence from sensing equipment distributed allover the network [14], [15].

\section{B. The OSMOSE Project}

OSMOSE (Optimal System-Mix Of Flessibility Solutions for European Electricity) is a $\mathrm{H} 2020$ project worth $28 \mathrm{M€}$, officially started in 2018 under the H2020 call "LCE-04-2017 - Demonstration of system integration with smart transmission grid and storage technologies with increasing share of renewables", involving 33 different European partners including several university departments, research centres, industrial partners, market players, and TSOs. Its main objective is to test new flexibility resources for the European power system. The project is divided into 8 Working Packages (WP), with several goals. Among these, the WP5, led by Terna, the Italian TSO, aims to assess and improve the techno-economic efficiency of flexibility services provision through a smart Zonal - Energy Management System (Z-EMS) by coordinating DTR sensors and algorithms, DSR by large industrial customer and RES generation. The demo area is a $380-220-150 \mathrm{kV}$ grid portion in the South of Italy.

\section{Contribution}

The Z-EMS developed within the OSMOSE project is a short term congestion management tool based on the coordinated exploitation of flexibility services provided by DSR and RES resources, and DTR limits of existing interconnections. In this sense, the Z-EMS is the first practical large scale application of optimal power flow techniques integrating DSR, RES and DTR technologies; its Technology Readiness Level is 8 , since it is being tested and validated in a real operational environment.

The scope of this paper is to provide insight on the mathematical formulation of the Z-EMS developed in the project and to provide first results of the ongoing real world experimentation.

\section{MAthematiCAL BACKGROUND}

This section provides the mathematical foundation of the proposed Z-EMS and an overview of its input data.

\section{A. Zonal Energy Management System Z-EMS Specifications}

The Z-EMS is based on a four-stage DC-OPF formulation, namely M1, M2, M3, and M4. Each stage covers different needs and their run is orchestrated in an ordered way. The main functionalities of these four models are:

M1 verifies the existence of a feasible power flow solution, within reasonable variation of the programmable generation and boundary buses foreseen balance; in other words, this stage acts as a test on the quality of the input data;

M2 checks whether congestion might happen in the future by solving power flow equations defined on the next 3 hours; 
M3 checks whether the congestion can be mitigated or solved by exploiting the knowledge of the dynamical thermal limits of the lines equipped with DTR technologies;

M4 checks whether the congestion might be mitigated or solved by exploiting the flexibility resources offered by the aggregators.

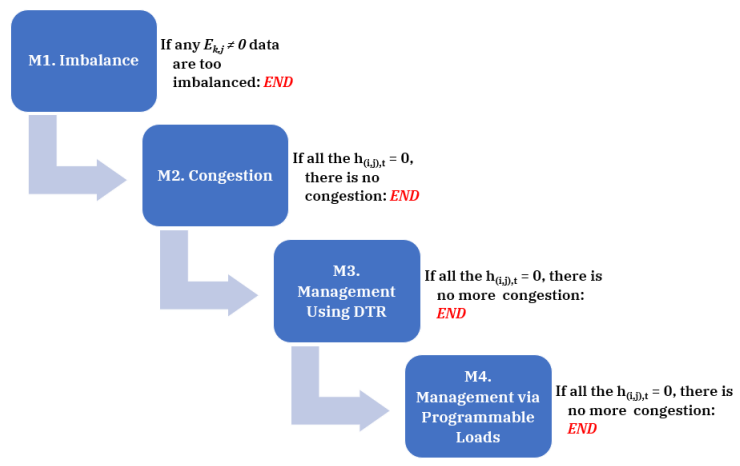

Fig. 1. Model Flow

Each stage is triggered by the successful execution of the previous one, according to the cascade flow logic specified in Fig. 1. All stages are based on DC-OPF formulations, which differ by small set of constraints, optimization variables and terms in the objective function. For the sake of brevity, only the M4 formulation (i.e. the most complete one) is described in the following.

$\min \mathrm{O}_{1}+\mathrm{O}_{2}+\mathrm{O}_{3}+\mathrm{O}_{4}$

s.t.:

$$
\begin{aligned}
\mathbf{P}_{t} & =\mathbf{B} \theta_{t} & & \\
P_{k, t} & =\hat{P}_{k, t}+\sum_{f \in \mathcal{F}_{k}^{p} \cup \mathcal{F}_{k}} \gamma_{f} \Delta p_{f, t}+ & & \\
& +\sum_{a \in\{g, b\}} \sum_{j \in\{k\} \cap \mathcal{N}^{a}} \Delta p_{j, t}^{a} & & \\
I_{(i, j), t} & +l_{(i, j)} \leq \hat{I}_{(i, j), t}^{u}+h_{(i, j), t}+h_{(i, j), t}^{\prime} & & \forall(i, j) \in \mathcal{L}(1 \mathrm{~d}) \\
h_{(i, j), t}^{\prime} & \leq \Delta \hat{I}_{(i, j), t}^{d} & & \\
-I_{(i, j), t} & \leq P_{(i, j), t} \leq I_{(i, j), t} & & \\
P_{(i, j), t} & =B_{(i, j), t}\left(\theta_{i, t}-\theta_{j, t}\right) & & \forall(i, j) \in \mathcal{L}(1 \mathrm{e}) \\
& & & \forall(i, j) \in \mathcal{L}(1 \mathrm{f})
\end{aligned}
$$

The objective function is composed of four terms:

$O_{1} \quad$ The $O_{1}$ part of the objective function aims at correctly 'driving' the power flow solution, by minimizing the sum of slack-to-target squares, that is keeping the active power balance at load, generation and boundary buses as much as possible close to the programs and forecast.

$$
\begin{aligned}
O_{1}= & \sum_{t \in \mathcal{T}}\left[c_{\Delta p}^{g} \sum_{k \in \mathcal{N}^{g}}\left(\Delta p_{k, t}^{g}\right)^{2}+c_{\Delta p}^{b} \sum_{k \in \mathcal{N}^{b}}\left(\Delta p_{k, t}^{b}\right)^{2}+\right. \\
& \left.+\sum_{f \in \mathcal{F}^{p}} \bar{\beta}_{f} \Delta p_{f, t}+c_{E} \sum_{k \in \mathcal{N}^{e}} E_{k, t}^{2}\right]
\end{aligned}
$$

$\mathrm{O}_{2} \quad$ Cost of the slack variables associated to the violation of STR line limits.

$$
O_{2}=\sum_{t \in \mathcal{T}} c_{h} \sum_{(i, j) \in \mathcal{L}} h_{(i, j), t}^{2}+c_{I} \sum_{(i, j) \in \mathcal{L}} I_{(i, j), t}
$$

$\mathrm{O}_{3} \quad$ Cost of the slack variables associated to the violation of DTR line limits.

$$
O_{3}=\sum_{k \in \mathcal{R}} c_{h^{\prime}} \sum_{(i, j) \in \mathcal{L}}\left(h_{(i, j), t}^{\prime}\right)^{2}
$$

$\mathrm{O}_{4}$ Cost for dispatching DSR resources: this is composed of an activation fee and a cost associated to the actual power deviation from the baseline.

$$
O_{4}=\sum_{t \in \mathcal{T}} c_{4} \sum_{f \in \mathcal{F}}\left(\bar{\beta}_{f} \Delta p_{f, t}+\bar{\alpha}_{f} \epsilon_{f, t}\right)
$$

The proposed framework is designed to exploit the flexibility offered by DSR resources only in case the updated DTR limits are not sufficient to mitigate the congestion. Moreover, the slack variables guarantee the feasibility of the optimization problem even in case the DSR resources are not able to solve the congestion. In this case, the congestion is still mitigated thanks to the coordinated exploitation of grid and DSR flexibility resources.

Because of the presence of binary variables, that model the activation signals for the DSR resources, and the quadratic terms in the objective function, the proposed formulation is a mixed-integer quadratic program, which can be solved by using commercial solvers, IBM CPLEX [16] in this specific case.

The congestion management process is based on the iterative solution of the framework shown in Fig. 1 on a moving time window. At each time step, the optimization procedure is repeated, based on an updated input data set (see Sec. II-B), and, if an action has to be taken, only the one corresponding to the first time instant is applied. This technique mitigates the error due to the uncertainty affecting the input data as the actions taken are constantly updated based on the latest available data. In the case of the Italian demo, a 3 hours - 15 minutes spaced moving time window has been chosen.

\section{B. Z-EMS Data}

The Z-EMS objective is to detect and solve congestion in the demo network within a 3 hours ahead time horizon. To achieve this goal, the Z-EMS exploits the following set of inputs.

- Current state of the grid - This is stored in two files, containing voltages, angles and power injections at each bus of the network and the admittance matrix. These two files are updated by Terna systems at each quarter of hour and then sent to the Z-EMS.

- Generation and Load forecast - For programmable generation the Z-EMS makes use of latest dispatching programs available, provided by Terna. Terna also provides forecast of non programmable generation and HVDC exchanges for the next 3 hours. Another software, named PREVEL, developed by RSE, provides the forecast of 
active power exchanges at each load and boundary bus of the network. Further details on PREVEL can be found in [17].

- DTR data - Two DTR algorithms have been developed and tested within the OSMOSE project, namely the sensor-based one and the weather-based one [13]. Both of them need weather forecast as input, but the former also needs measurements from weather sensors installed over the lines (seven, in the project). Both the DTR algorithms elaborate the loadability curve, i.e., the maximum current that can flow over the lines as a function of time.

- DSR data - In order to activate DSR resources, the Z-EMS needs to communicate with Balancing Service Providers (BSPs). In particular, BSPs send aggregated industrial offers to Z-EMS, that selects the best ones through the optimization procedure described in the previous section. Each BSP sends offers for only one fixed grid node, where all BSP industrial loads are aggregated.

While the first two sets of data are mandatory for each ZEMS run, the third and fourth ones are optional. In fact, if DTR and DSR data are not available, Z-EMS will be at least able to elaborate the congestion detection. All data exchanges are handled by a software, namely the Hand of Data, which collects all input and output data of the Z-EMS for the current 15 minutes and saves them in an appropriate repository.

\section{Z-EMS DEMONSTRATOR PRELIMINARY RESULTS}

The Z-EMS is currently undergoing its experimental phase, which started in March 2021 and is expected to end in October 2021. During this period, the Z-EMS is running in parallel with Terna network control center tools in order to guarantee a stable, continuous and safe operation of the system. For this reason, the mitigation actions proposed by the Z-EMS are not directly implemented, but require the manual activation by the control center operators. This also means that, in general, it is unlikely to incur in network congestion, as the Terna control systems already act to prevent them. Hence, in order to assess the capability of the model in detecting and mitigating the congestion, fictitious limits are imposed on a selected set of lines. In other words, congestion are simulated, so that the ZEMS can detect them and propose some corrective actions to the control center operators.

To get a feeling of the model ability to predict the congestion, and their possible mitigation, it is necessary to assess the quality of the grid state prediction. In practice, this consists in evaluating the impact of the following assumptions of the model:

1) The DCOPF formulation is missing the $Q$ value. To overcome this limitation, the $Q$ values from the input data at $t_{0}$ are persisted along the entire time horizon. This persistence is a good proxy during the first steps, but the predictive power decays sharply after the first hour.

2) The network is topologically unchanged during the 12 time steps $\left(t_{1}, \ldots, t_{12}\right)$. This assumption frequently

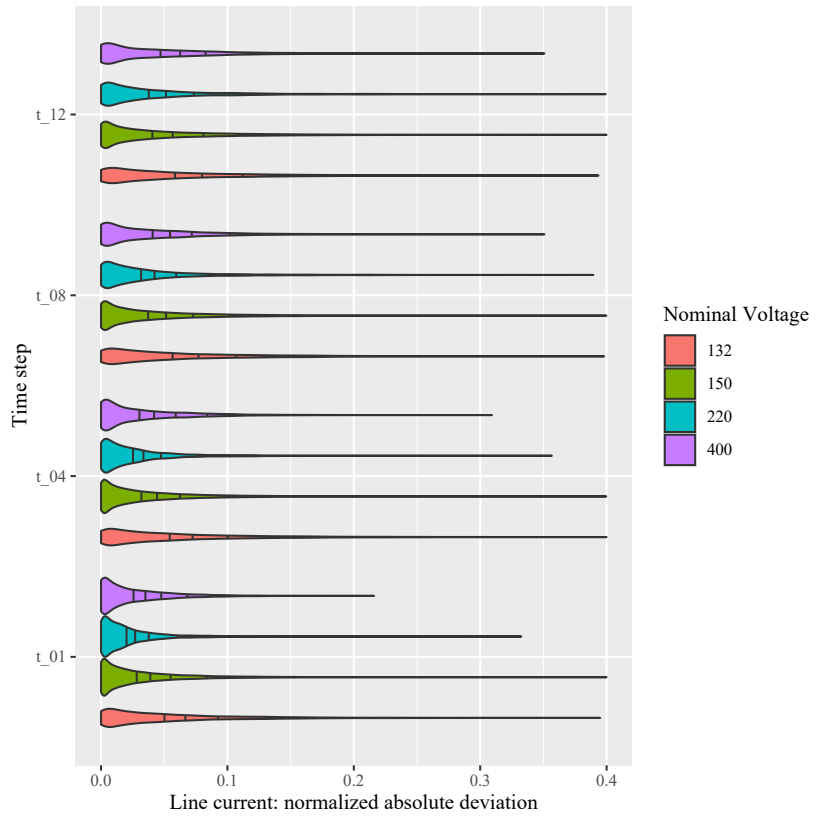

Fig. 2. Absolute Deviation of current estimation in selected time steps. The lines within the 'violin' represent the 60th, 70th, 80th, and 90th percentiles.

doesn't hold in practice, as the incidence matrix or lines admittance values might change.

3) The forecast of loads and generation is affected by uncertainty.

In order to test the discussed assumptions, the deviations at a line/time step level over all the Z-EMS run in July 9th 2021 are analyzed; Fig. 2 shows the distribution of the deviations from the field measurements at line/nominal voltage level, in four time steps, i.e., time step $=1,4,8,12$ at $15^{\prime}, 60^{\prime}, 120^{\prime}, 180^{\prime}$, respectively. All deviations are normalized as a fraction of the current limit set for the line. As expected, the quantile deviations increase together with time steps; however, within the first hour, nearly $80 \%$ of the lines have a predicted current within $5 \%$ of the field measurements. However, this analysis confirms that the uncertainty on load and generation forecasts does not significantly affect the line current deviations. It is also worth noting that the normalized deviation is also smaller on the higher voltage part of the network.

On the other hand, a strong and significant correlation between the normalized absolute deviation, and the number of changes in the network topology has been observed. The changes in network topology have been measured simply by summation, at each time step, of the number of lines or buses, which were present at $t_{0}$ but were not listed at $t_{n}$, and vice versa. The behavior of the median and of the 90th percentile of the normalized deviation, given the \# of topology changes, are shown in Fig. 3 and Fig. 4, respectively. This correlation is significant but it could be due to the effect of the distinct $t_{n}$ (i.e. it could also be due to the error induced by the $Q_{t_{0}}$ persistence). To rule out this effect, the correlation significance at each time step is separately checked. In the first hour the 


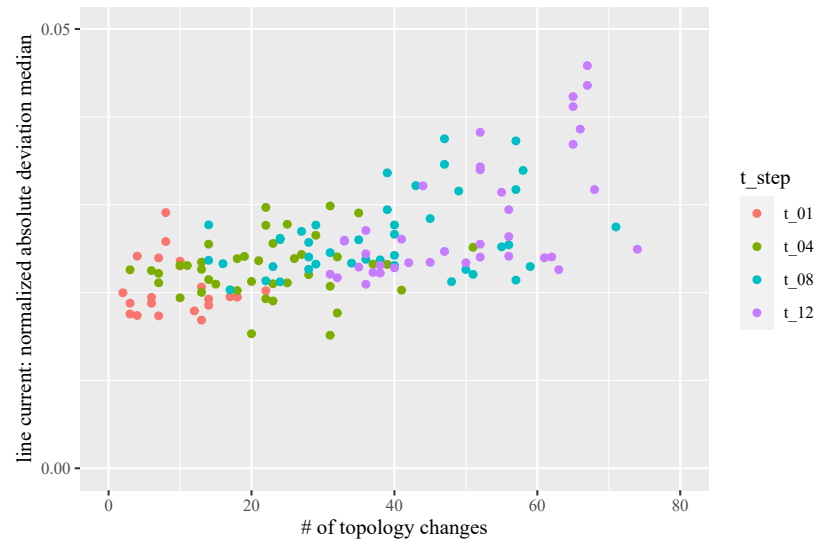

Fig. 3. Relationship between median absolute deviation of current estimation and topology changes in the grid.

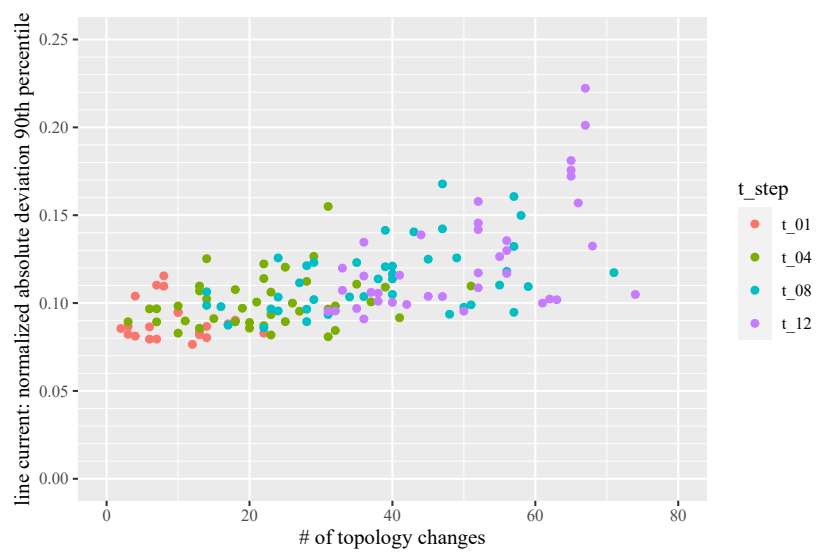

Fig. 4. Relationship between 90th percentile of absolute deviation of current estimation and topology changes in the grid.

correlation is not significant and this might be attributed to the small variability of \# of changes, especially in $t_{1}$, while it is significant $(p<0.001)$ in $t_{8}$ and $t_{12}$.

These results demonstrate that most of the lines have a predicted current within a deviation error that allows to predict most of the large congestion, thus confirming the validity of the approach.

\section{CONCLUSIONS AND FUtURE WORK}

In this paper, the mathematical formulation of the Z-EMS developed in the EU OSMOSE project has been presented, and some first experimentation results have been discussed. So far, Z-EMS has ran for three months with real-time data and with full DTR functionalities, while, in the next months, DSR functionalities will be tested. The first results of the experimentation confirm the validity of the overall framework proposed in the project, as the Z-EMS proves to be able to correctly predict the state of the network with a reasonable confidence level, despite the forecast uncertainties and model approximations considered.

The experimental results also pave the way to possible enhancements of the Z-EMS itself. Moving towards a full
ACOPF implementation seems feasible as far as computational times, but will require the availability of forecasts also on the reactive power injections at each bus. Moreover, more information on the future decisions on the network topology, as well as the near real time decision on some programmable generators, could further improve the ability to detect any congestion.

\section{REFERENCES}

[1] International Energy Agency, "World energy outlook 2019," International Energy Agency, 2019.

[2] A. Baczyńska and W. Niewiadomski, "Power flow tracing for active congestion management in modern power systems," Energies, vol. 13, no. 18 , p. $4860,2020$.

[3] P. Wei, R. Fu, Y. Lu, Q. Wang, L. Wang, Y. Li, and G. Tang, "Congestion-based model for transmission expansion planning," in 2006 IEEE Power Engineering Society General Meeting. IEEE, 2006, pp. 6-pp.

[4] H. Saberi, H. Monsef, and T. Amraee, "Probabilistic congestion driven network expansion planning using point estimate technique," IET Generation, Transmission \& Distribution, vol. 11, no. 17, pp. 4202-4211, 2017.

[5] K. Verma, S. Singh, and H. Gupta, "Location of unified power flow controller for congestion management," Electric power systems research, vol. 58, no. 2, pp. 89-96, 2001.

[6] S. Singh and A. David, "Optimal location of facts devices for congestion management," Electric Power Systems Research, vol. 58, no. 2, pp. 7179, 2001.

[7] S. Dutta and S. Singh, "Optimal rescheduling of generators for congestion management based on particle swarm optimization," IEEE transactions on Power Systems, vol. 23, no. 4, pp. 1560-1569, 2008.

[8] J. R. Chintam and M. Daniel, "Real-power rescheduling of generators for congestion management using a novel satin bowerbird optimization algorithm," Energies, vol. 11, no. 1, p. 183, 2018.

[9] S. Verma, S. Saha, and V. Mukherjee, "Optimal rescheduling of real power generation for congestion management using teaching-learningbased optimization algorithm," Journal of Electrical Systems and Information Technology, vol. 5, no. 3, pp. 889-907, 2018.

[10] I. Liere-Netheler, F. Schuldt, K. von Maydell, and C. Agert, "Optimised curtailment of distributed generators for the provision of congestion management services considering discrete controllability," IET Generation, Transmission \& Distribution, vol. 14, no. 5, pp. 735-744, 2020.

[11] F. Zaeim-Kohan, H. Razmi, and H. Doagou-Mojarrad, "Multi-objective transmission congestion management considering demand response programs and generation rescheduling," Applied Soft Computing, vol. 70, pp. 169-181, 2018.

[12] P. Sharmila, J. Baskaran, C. Nayanatara, S. G. CS, and H. N. C, "Congestion management in deregulated power systems using genetic algorithm," in 2020 International Conference on Power, Energy, Control and Transmission Systems (ICPECTS), 2020, pp. 1-9.

[13] D. Villacci, F. Gasparotto, L. Orrú, P. Pelacchi, D. Poli, A. Vaccaro, G. Lisciandrello, and G. Coletta, "Congestion Management in Italian HV grid using novel Dynamic Thermal Rating methods: first results of the H2020 European project OSMOSE," in 2020 AEIT International Annual Conference (AEIT), 2020, pp. 1-6.

[14] G. Coletta, A. Laso, G. M. Jónsdóttir, M. Manana, D. Villacci, A. Vaccaro, and F. Milano, "On-line control of ders to enhance the dynamic thermal rating of transmission lines," IEEE Transactions on Sustainable Energy, vol. 11, no. 4, pp. 2836-2844, 2020.

[15] N. Viafora, K. Morozovska, S. H. H. Kazmi, T. Laneryd, P. Hilber, and J. Holbøll, "Day-ahead dispatch optimization with dynamic thermal rating of transformers and overhead lines," Electric Power Systems Research, vol. 171, pp. 194-208, 2019.

[16] IBM, User's Manual for CPLEX. IBM Corp, 2020. [Online]. Available: https://www.ibm.com/docs/en/icos/20.1.0?topic=cplex-users-manual

[17] D. Ronzio, E. Collino, G. Lisciandrello, and L. Orrù, "An innovative algorithm for the power loads forecasting in italian transmission grid: development and main results of the prevel software of osmose project," in Submitted to 2021 AEIT International Annual Conference (AEIT), 2021. 